\title{
A NOTE ON SECOND SPECIES SOLUTIONS GENERATED FROM $p-q$ RESONANT ORBITS
}

\author{
ESTHER BARRABÉS ${ }^{1}$ and GERARD GÓMEZ ${ }^{2}$ \\ ${ }^{1}$ Departament d'Informàtica i Matemàtica Aplicada, Universitat de Girona, \\ Av. Lluís Santaló, s/n, 17071 Girona, Spain,e-mail: barrabes@ima.udg.es \\ ${ }^{2}$ Departament de Matemàtica Aplicada i Anàlisi, Universitat de Barcelona, IEEC, \\ Gran Via 585, 08007 Barcelona, Spain, e-mail: gerard@maia.ub.es
}

(Received: 9 May 2003; revised: 27 August 2003; accepted 2 September 2003)

\begin{abstract}
The first image under the flow of the restricted three-body problem of the $p-q$ resonant strips - that appear in the study of the $p-q$ resonant orbits - do not have, in general, intersection with the strip. In this paper we show some particular situations in which the above intersections exist for some very simple $p-q$ resonant orbits which, at the same time, are periodic second species solutions.
\end{abstract}

Key words: periodic orbits, restricted three-body problem, second species solutions

\section{Introduction}

In this paper we will study the circular restricted three-body problem (RTBP) with the usual units of mass, length and time. $E$ and $M$ will denote the two primaries, with masses $1-\mu$ and $\mu$, respectively. Since we will consider small values of $\mu$, $E$ will be referred as the big primary and $M$ as the small one.

The $p-q$ resonant orbits are trajectories of the infinitesimal body, $P$, which, between two consecutive close approaches to the small primary, perform $p$ revolutions around $E$ while $M$ does $q$ revolutions around the centre of masses of the primaries. More precisely, the time spent by these orbits after leaving the ball $B\left(M, \mu^{\alpha}\right)$ in the configuration space, with centre at $M$ and radius $\mu^{\alpha}$, and returning for the first time to the same ball is

$$
T=2 \pi q+\varepsilon \mu^{\alpha}+O\left(\mu^{2 \alpha}\right)=2 \pi \tau p+\delta \mu^{\alpha}+O\left(\mu^{2 \alpha}\right),
$$

where $p, q \in \mathbb{N}$ are relatively prime, $\epsilon$ and $\delta$ are suitable constants and $2 \pi \tau$ is the period of the elliptic orbit which approaches the motion of $P$ around $E$, neglecting the effects of $M$. In the above expressions $\alpha$ is a suitable small real parameter. A solution of the RTBP obtained gluing together several resonant orbits is called a consecutive quasi-collision orbit. These orbits are a generalization of the so-called orbits with consecutive collisions, which have been studied in Hénon $(1968,1997)$ for the circular RTBP and in Gómez and Olle (1986) for the elliptic case. An orbit 


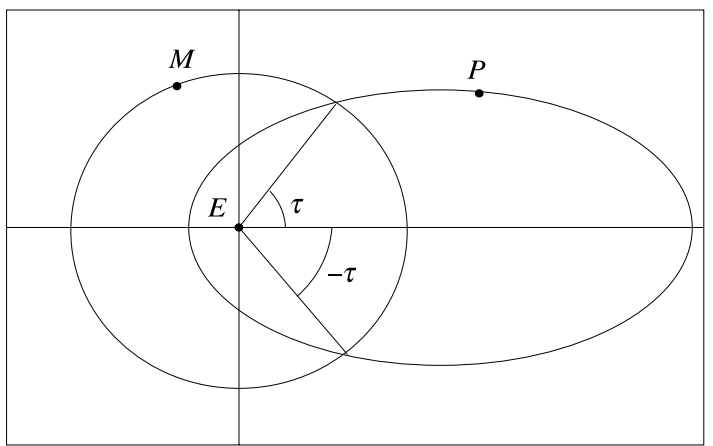

Figure 1. Orbits of consecutive collisions: $P$ (with elliptic motion) meets $M$ (with circular motion and $\mu=0$ ) at epochs $-\tau$ and $\tau$.

of the RTBP, with $\mu=0$, is said to be of consecutive collisions if it meets $M$ at two epochs, $-\tau$ and $\tau$, as is shown in Figure 1. When a consecutive quasi-collision orbit is periodic then, according to Poincaré (1899), is called a second species solution (SSS). Orbits with consecutive collisions are the natural limit (when $\mu \rightarrow 0$ ) of second species solutions.

The planar $p-q$ resonant orbits have been first studied by Font et al. (2002). They define the resonant strips as the set of initial conditions on $B\left(M, \mu^{\alpha}\right)$ which correspond to $p-q$ resonant orbits and study the behaviour of these strips, under the flow associated to the RTBP, in the following way: given an initial conditions on $B\left(M, \mu^{\alpha}\right)$ they consider its image under the flow until the second intersection with the sphere (from the initial conditions until the first intersection, the orbit moves outside the sphere and from this point until the second intersection, inside it). The study of the map shows that, if the mass parameter $\mu$ is small enough, it behaves like a 'horseshoe'-map, so there exist infinitely many consecutive quasicollision periodic orbits (SSSs), corresponding to periodic sequences $\left(p_{n}, q_{n}\right)_{n \in Z}$. Nevertheless, from the numerical results of Font et al. (2002) it seems that, in general, the first image of a resonant strip under the composition of both maps, does not intersect the resonant strip; this should give the simplest SSSs of the RTBP. In the present study we will focus on this kind of solutions.

The interest in the study of this kind of solutions comes from their application to the design of spacecraft missions, since they can be used to reduce the orbit capture $\Delta V$ (Yen, 1985), and also because of their role as natural motion of comets. For example, the comet Oterma moves from a heliocentric orbit outside the orbit of Jupiter to an orbit inside that of Jupiter. The interior orbit is near a 3:2 resonance while the exterior orbit is near a 2:3 resonance, see Koon et al. (2001). This process can be described as a hopping from one resonance to another as is discussed in Belbruno and Marsden (1997).

The purpose of this paper is to study which $p-q$ resonant orbits can be extended to periodic SSSs of the RTBP. For this goal we will use the analytic asymptotic approximations of the $p-q$ resonant orbits obtained in Barrabés and Gómez (2002; 
2003) for the three-dimensional RTBP, although we will focus our attention to the planar case. In order to obtain these approaches, we have studied the so-called out-map and in-map, which are defined as follows: consider initial conditions on $B\left(M, \mu^{\alpha}\right)$ and the return map to the same sphere under the flow associated to the RTBP. The out-map is associated to follow the flow forwards (and far from the small primary) and the in-map to follow the flow backwards (inside $B\left(M, \mu^{\alpha}\right)$ ). Using the analytical expansions (in powers of the mass parameter $\mu$ ) of the outmap and in-map, and matching these expressions, a set of initial conditions of orbits that are close to periodic with an error of order $\mu^{1-\alpha}$, is obtained. These are the orbits that we will try to extend numerically to periodic orbits.

The paper is organized as follows: Section 2 is a short survey of the results related to the initial conditions of the 'periodic' orbits (up to $\mu^{1-\alpha}$ order) under consideration. In Section 3 we give the results of the numerical explorations corresponding to the refinement of the initial conditions obtained in Section 2. Finally, in Section 4 we perform the numerical continuation (for $\mu>0$ ) of some families of orbits with consecutive collisions and identify the periodic orbits obtained in Section 3 on these families.

\section{Initial Conditions of the $p-q$ Resonant Orbits Close to SSS}

Let $\mathbf{r}_{0}=\left(x_{0}, y_{0}\right)$ and $\dot{\mathbf{r}}_{0}=\left(\dot{x}_{0}, \dot{y}_{0}\right)$ be the initial conditions (without loss of generality we can suppose they are at epoch $t=0$ ) of a planar $p-q$ resonant orbit in a synodical reference system, in which $M$ is located at $\mathbf{r}_{M}=(\mu-1,0)$. Let us suppose that $P$ leaves a circle $\mathrm{C}$ of radius $\mu^{\alpha}$ around the small primary, $M$, moving away from it. The initial conditions can be written as

$$
\begin{aligned}
& x_{0}=\mu-1+\mu^{\alpha} \cos \theta, \quad y_{0}=\mu^{\alpha} \sin \theta, \quad \dot{x}_{0}=v \cos \bar{\psi}, \\
& \dot{y}_{0}=v \sin \bar{\psi},
\end{aligned}
$$

and they must verify the condition

$$
\epsilon \leqslant \frac{\left\langle\mathbf{r}_{0}-\mathbf{r}_{M}, \dot{\mathbf{r}}_{0}\right\rangle}{\mu^{\alpha} v}
$$

which ensures that we are avoiding the tangent direction. For a fixed position on $\mathrm{C}$, there are different velocities for which the orbit comes back to the same circle. Thus, we write $\bar{\psi}=\psi+\Delta \psi \mu^{\alpha}+O\left(\mu^{2 \alpha}\right)$ and condition (3) can be written as $\epsilon \leqslant \cos (\psi-\theta)$, for some $\epsilon$.

Let $\left(\mathbf{r}_{e}, \dot{\mathbf{r}}_{e}\right)$ be the image of $\left(\mathbf{r}_{0}, \dot{\mathbf{r}}_{0}\right)$ under the out-map, this is, the position and velocity vectors obtained following the flow forward from the initial conditions until $t=T$ (defined by (1)). Analogously, let $\left(\mathbf{r}_{i}, \dot{\mathbf{r}}_{i}\right)$ be the image of $\left(\mathbf{r}_{0}, \dot{\mathbf{r}}_{0}\right)$ by the in-map, this is, the position and velocity vectors obtained following the flow backward inside $\mathrm{C}$ until the orbit cross the circle again. An analytical developments 
for both maps can be given in terms of $\mu^{\alpha}$ (for the computation of both maps see Barrabés and Gómez $(2002,2003)$. From the definition of a $p-q$ resonant orbit and matching these expressions up to order $\mu^{\alpha}$, we obtain conditions on $\theta, \psi, v$ and the corresponding Jacobi constant $C_{J}$ which ensure that the orbit is a $p-q$ resonant orbit close to a SSS.

The restrictions are the following. For each $p, q \in \mathbb{N}$ relatively prime with $p / q \leqslant 2 \sqrt{2}$, there is a family of $p-q$ resonant orbits with the Jacobi constant, $C_{J}$, within the interval

$$
\begin{aligned}
C_{J_{1}} & =\left(\frac{p}{q}\right)^{2 / 3}-2 \sqrt{2-\left(\frac{p}{q}\right)^{2 / 3}} \\
& <C_{J}<\left(\frac{p}{q}\right)^{2 / 3}+2 \sqrt{2-\left(\frac{p}{q}\right)^{2 / 3}}=C_{J_{2}} .
\end{aligned}
$$

The value $(p / q)^{2 / 3}$ should be excluded from this interval because it corresponds to a collision orbit in the two-body problem. For each value of $C_{J}$ within the above set, there are two admissible values of the polar angle of the velocity, $\psi$, given by the relation

$$
\sin \psi=\frac{2-C_{J}+(p / q)^{2 / 3}}{2 \sqrt{3-C_{J}}} .
$$

In Figure 2 we have represented some of the curves $C_{J}(\psi)$ defined by (5) for different values of $p$ and $q$. As it can be seen, when $p>q$ the curves are closed, while if $p<q$ they are open. In all the cases they are symmetric with respect to the $\psi=\pi / 2$ axis.

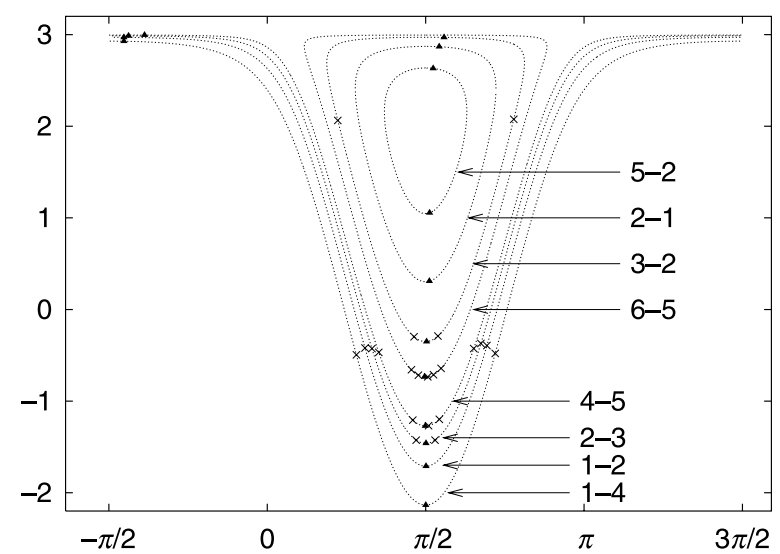

Figure 2. Behaviour of the polar angle $\psi$ ( $x$-axis) as a function of the Jacobi constant ( $y$-axis) for the values of $(p, q)=(1,4),(1,2), \ldots,(5,2)$. For further comments on this figure see Section 3 . 
Finally, for every admissible pair $\left(C_{J}, \psi\right)$, there exists one value for $\theta$ such that the initial conditions (2) satisfy the corresponding matching conditions: $\mathbf{r}_{e}=\mathbf{r}_{i}$ and $\dot{\mathbf{r}}_{e}=\dot{\mathbf{r}}_{i}$. This value is the solution of the equation

$$
\frac{2}{\sqrt{3-C_{J}}}\left(\cos \theta \sin ^{2}(\theta-\psi)-\cos \psi \cos (\theta-\psi)\right)+\sin ^{3}(\theta-\psi)=0,
$$

such that $\cos (\theta-\psi)>0$, in order to satisfy (3).

Moreover, one can compute the total time required to come back close to the initial condition (2), which is

$$
\begin{aligned}
T & =\left(2 \pi q+\epsilon \mu^{\alpha}+O\left(\mu^{2 \alpha}\right)\right)+\left(\frac{2 \cos (\theta-\psi)}{\sqrt{3-C_{J}}} \mu^{\alpha}+O\left(\mu^{2 \alpha}\right)\right) \\
& =2 \pi q+O\left(\mu^{\alpha}\right) .
\end{aligned}
$$

All these magnitudes, initial conditions and $T$, will be taken as a first approximation of the initial conditions and period of the periodic second species solutions close to $p-q$ resonant orbits.

\section{Numerical Refinement of the $p-q$ Resonant Orbits}

Using the initial conditions of the $p-q$ resonant orbits given in the preceding section, we have proceeded to its numerical refinement in order to find periodic orbits. We have used a very small value of the mass ratio, $\mu=10^{-6}$, in order to start with a good analytic approximation. According to Font et al. (2002), only a very reduced subset of these orbits will be periodic, if we ask to the period to be close to the value of $T$ given by (7). This is the reason why, in principle, we have just asked for periodicity in the configuration space, so, at the end of the integration there will be a small difference, $\Delta v$, between the initial and final velocities.

First of all, we will see that we can use the standard implicit function theorem in order to obtain periodic orbits in the configuration space. Let us see the equations involved. The first two equations come from the fact that we want a $p-q$ resonant orbit so, from its definition, we have that

$$
\tau=\frac{p}{q}+\frac{\varepsilon-\delta}{2 \pi p} \mu^{\alpha}+O\left(\mu^{2 \alpha}\right) \quad \text { and } \quad\left|\mathbf{r}_{e}-\mathbf{r}_{M}\right|=\mu^{\alpha},
$$

which can be expressed in terms of the initial conditions as follows:

$$
\begin{aligned}
f_{1} \equiv & C_{J}-2+2 \sqrt{3-C_{J}} \sin \psi-\left(\frac{p}{q}\right)^{2 / 3}+O\left(\mu^{\alpha}\right)=0 \\
f_{2} \equiv & (\varepsilon-\delta)^{2}+\delta^{2}\left(3-C_{J}\right)+2(\varepsilon-\delta) \sin \theta+ \\
& +2 \delta \sqrt{3-C_{J}}(\cos (\psi-\theta)+(\varepsilon-\delta) \sin \psi)+O\left(\mu^{1-2 \alpha}\right)=0 .
\end{aligned}
$$


The other two equations ensure the periodicity in the configuration space:

$$
x_{e}=x_{i}, \quad y_{e}=y_{i},
$$

where $\mathbf{r}_{e}=\left(x_{e}, y_{e}\right)$ and $\mathbf{r}_{i}=\left(x_{i}, y_{i}\right)$, and they can be written as

$$
\begin{aligned}
& f_{3} \equiv\left(\delta \sqrt{3-C_{J}}+2 \cos (\psi-\theta)\right) \cos \psi+O\left(\mu^{\alpha}\right)=0, \\
& f_{4} \equiv(\varepsilon-\delta)+\left(\delta \sqrt{3-C_{J}}+2 \cos (\psi-\theta)\right) \sin \psi+O\left(\mu^{\alpha}\right)=0 .
\end{aligned}
$$

Let us see that it will be enough to deal with $f_{1}=0, f_{2}=0$ and $f_{3}=0$. From the definition of the inner map, we have that $\left|\mathbf{r}_{i}-\mathbf{r}_{M}\right|=\mu^{\alpha}$, so

$$
\left.\begin{array}{c}
\left|\mathbf{r}_{e}-\mathbf{r}_{M}\right|=\mu^{\alpha}, \\
x_{e}=x_{i},
\end{array}\right\} \Rightarrow y_{e}^{2}=y_{i}^{2} \Rightarrow y_{e}= \pm y_{i} .
$$

On the other hand, let $\zeta=(\theta, \psi, \varepsilon-\delta, \delta)$ and $F=F(\zeta, \mu)=\left(f_{1}, f_{2}, f_{3}\right)$. Suppose that there exists $\zeta_{0}$ such that $F\left(\zeta_{0}, 0\right)=0$ and $y_{i}\left(\zeta_{0}\right)=y_{e}\left(\zeta_{0}\right)$, and a continuous family $\zeta(\mu)$ such that $F(\zeta(\mu), \mu)=0$ and $\zeta(0)=\zeta_{0}$. Then $y_{i}(\zeta(\mu))=y_{e}(\zeta(\mu))$ for some $\mu$.

Finally, let us see that we can apply the implicit function theorem to the equation $F(\zeta, \mu)=0$. For every $p, q$ and $C_{J}$ verifying (4), there is a solution $\zeta_{0}$ of $F(\zeta, 0)=0$, such that satisfy $f_{4}=0$, given by

$$
\varepsilon-\delta=0, \quad \delta=\frac{-2 \cos (\psi-\theta)}{\sqrt{3-C_{J}}},
$$

and $\psi$ and $\theta$ verifying (5) and (6). Next, the matrix of partial derivatives respect to $\zeta$ evaluated on these solutions is

$$
D F\left(\zeta_{0}, 0\right)=\left(\begin{array}{cccc}
0 & f_{12} & 0 & 0 \\
f_{21} & f_{22} & f_{23} & f_{24} \\
f_{31} & f_{32} & 0 & f_{34}
\end{array}\right),
$$

where

$$
\begin{aligned}
& f_{12}=f_{34}=2 \sqrt{3-C_{J}} \cos \psi, \quad f_{21}=-f_{22}=-4 \cos (\psi-\theta) \sin (\psi-\theta), \\
& f_{31}=-f_{32}=2 \sin (\psi-\theta) \cos \psi, \quad f_{24}=-2 \sqrt{3-C_{J}} \cos (\psi-\theta), \\
& f_{23}=2 \sin \theta-4 \cos (\psi-\theta) \sin \psi,
\end{aligned}
$$

and the determinant of the last three columns is equal to

$$
\begin{aligned}
& 2\left(3-C_{J}\right) \cos ^{2} \psi(2 \sin \theta-4 \cos (\psi-\theta) \sin \psi) \\
& \quad=-4\left(3-C_{J}\right) \cos ^{2} \psi \sin (2 \psi-\theta) .
\end{aligned}
$$

From (4), $C_{J} \neq 3$ and, combining with (5), $\cos \psi \neq 0$. Then, if $\theta \neq 2 \psi-k \pi$, the implicit function theorem can be applied: given $\zeta_{0}$ defined above, for $\mu>0$ there exists $\zeta(\theta, \mu)$ such that $F(\zeta(\theta, \mu), \mu)=0$ and $\zeta(\theta, 0)=\zeta_{0}$. 

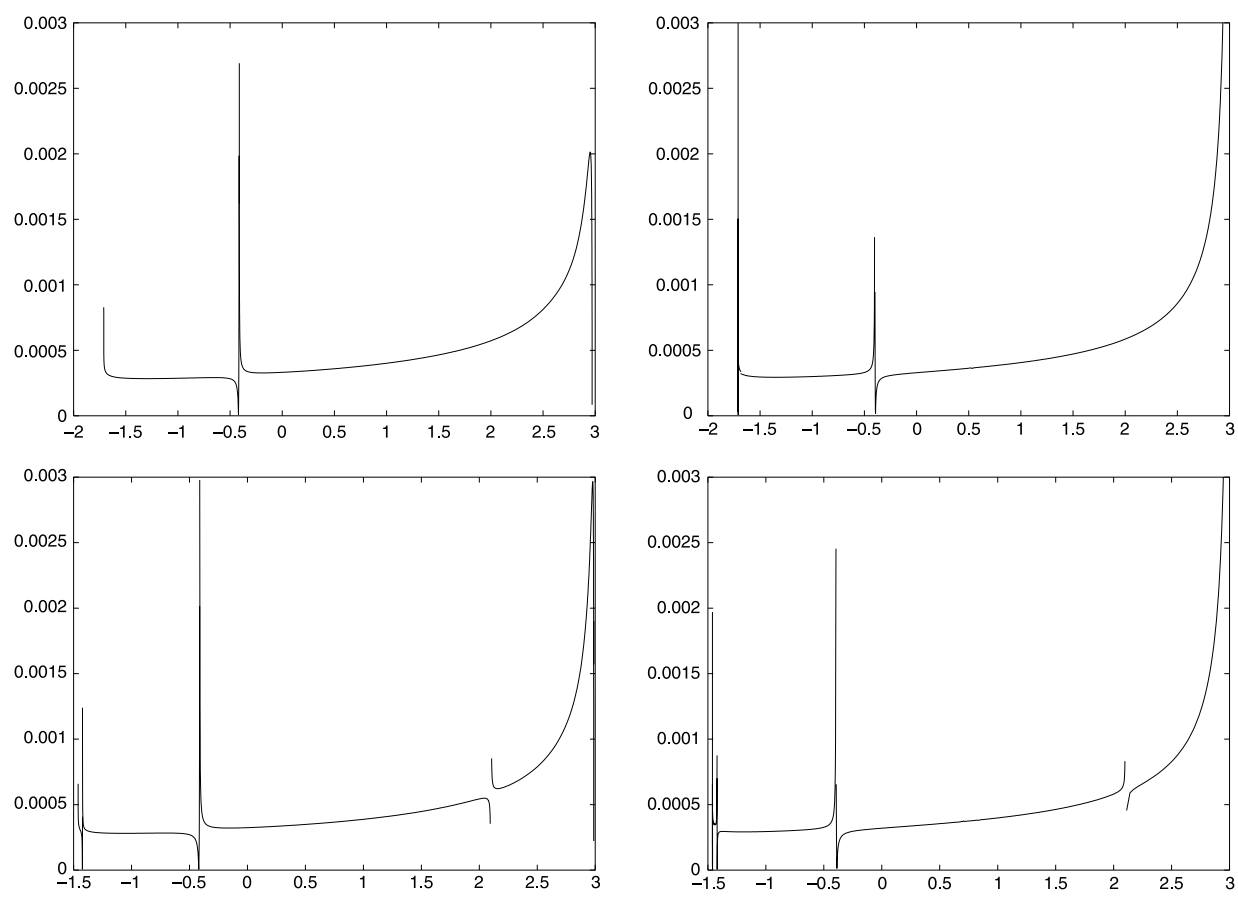

Figure 3. $C_{J}$ ( $x$-axis) versus $\Delta v$ ( $y$-axis) for the orbits of the $p-q$ resonant families with $p<q$. The left-hand side curves correspond to values of $\psi \in(-\pi / 2, \pi / 2)$ and the right-hand side to $\psi \in(\pi / 2,3 \pi / 2)$. From top to bottom, the values of $p-q$ are $1-2$ and $2-3$ respectively.
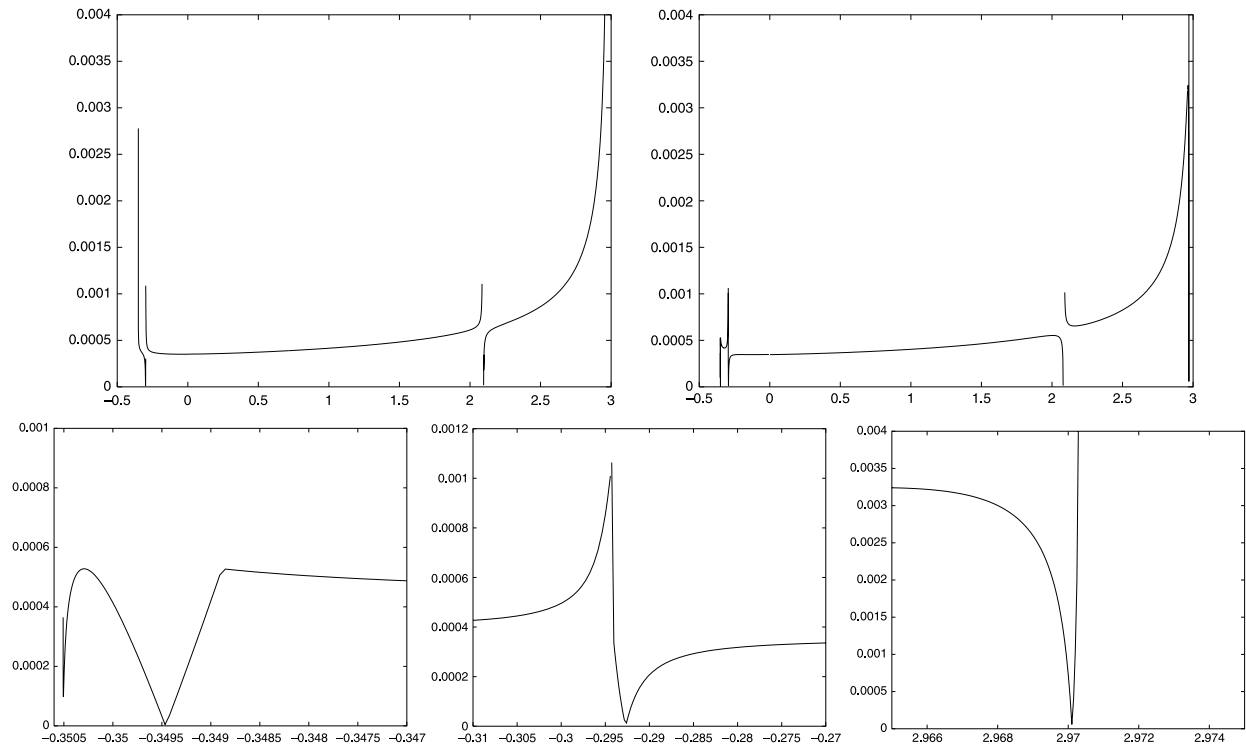

Figure 4. $C_{J}$ ( $x$-axis) versus $\Delta v$ (y-axis) for the orbits of the 3-2 resonant families. At the top, the left-hand side curves correspond to values of $\psi \in(-\pi / 2, \pi / 2)$ and the right-hand side to $\psi \in(\pi / 2,3 \pi / 2)$. At the bottom, the figures are details of the right-hand side curve. 
TABLE I

For several values of $p-q$ with $p<q$, Jacobi constant, period and initial conditions of the periodic orbits computed (for all the orbits $y_{i}=0$ )

\begin{tabular}{rlrlrrr}
\hline$p-q$ & No. & \multicolumn{1}{l}{$C_{J}$} & Period & \multicolumn{1}{c}{$x_{i}$} & \multicolumn{1}{c}{$\dot{x}_{i}$} & $\dot{y}_{i}$ \\
\hline $1-2$ & 1 & 2.970310637789 & 12.528640250692 & -1.243599633902 & 0.304685981834 & 0.302714065050 \\
& 2 & -0.419841452236 & 12.566038319741 & -1.004211162190 & 1.043407035798 & 1.526979732499 \\
& 3 & -1.710974993097 & 12.570221966265 & -0.999241351069 & 0.000000000018 & 2.171085549004 \\
& 4 & -1.709952533642 & 12.567874800223 & -0.998053329474 & 0.000000000015 & 2.170481013177 \\
& 5 & -0.393793552693 & 12.566707243934 & -0.995769395386 & -1.055469239756 & 1.510066526569 \\
$1-4$ & 1 & 2.927891220262 & 25.120782877589 & -0.998677046177 & -0.000000000506 & -0.271335473533 \\
& 2 & -0.497138413902 & 25.132311181853 & -1.004347740058 & 1.181819460726 & 1.449466677880 \\
& 3 & -2.135454263704 & 25.139933288244 & -0.999741414754 & 0.0000000000005 & 2.267865710897 \\
& 4 & -2.133739275587 & 25.133753341565 & -0.998159294719 & 0.000000000051 & 2.266016895845 \\
& 5 & -0.476954817361 & 25.133176417736 & -0.995637826084 & -1.191241999340 & 1.434715698175 \\
& 1 & 2.986969746680 & 18.765356000879 & -1.157618109612 & 0.204738988149 & 0.197197456855 \\
& 2 & -0.420143435496 & 18.849297495181 & -1.004135937321 & 0.938988531693 & 1.593416023510 \\
& 3 & -1.424134420615 & 18.847772700544 & -1.003073873895 & 0.204547791866 & 2.093554241284 \\
& 4 & -1.461178013314 & 18.854177064908 & -0.999037568288 & 0.000000000015 & 2.112642189083 \\
& 5 & -1.460482256304 & 18.851741835592 & -0.997965460689 & -0.000000000004 & 2.112220202991 \\
& 6 & -1.420084405637 & 18.851341555496 & -0.996915333448 & -0.210246852473 & 2.092021470020 \\
& 7 & -0.387412938178 & 18.849825622298 & -0.995839311492 & -0.954590193929 & 1.573753271199 \\
\hline
\end{tabular}


TABLE II

For several values of $p-q$ with $p>q$, Jacobi constant, period and initial conditions of the periodic orbits computed (for all the orbits $y_{i}=0$ )

\begin{tabular}{lllllll}
\hline$p-q$ & No. & \multicolumn{1}{l}{$C_{J}$} & \multicolumn{1}{l}{ Period } & \multicolumn{1}{c}{$x_{i}$} & \multicolumn{1}{c}{$\dot{x}_{i}$} & $\dot{y}_{i}$ \\
\hline $2-1$ & 1 & 0.305459446447 & 6.283875243979 & -1.001904853601 & -0.000000000007 & 1.641827285408 \\
& 2 & 2.868215132962 & 6.280311511416 & -1.002173873302 & -0.000000000047 & 0.364300192862 \\
$3-2$ & 1 & 2.096417809899 & 12.567702458789 & -0.994620541732 & 0.737740084019 & 0.599813894926 \\
& 2 & -0.299241734918 & 12.565171131410 & -0.997047808483 & 0.208849852158 & 1.804528566326 \\
& 3 & -0.350178252237 & 12.568468261691 & -1.001380576489 & 0.000000000004 & 1.830745092811 \\
& 4 & -0.350389788445 & 12.568742448535 & -1.001222252191 & 0.000000000002 & 1.830853696956 \\
& 5 & -0.349483371265 & 12.567807755595 & -1.002007108787 & -0.000000000006 & 1.830433668445 \\
& 6 & -0.292688569206 & 12.567513588044 & -1.002995509853 & -0.213059303221 & 1.802216587767 \\
& 7 & 2.078912548584 & 12.565075793271 & -1.005305130404 & -0.730069620860 & 0.623332086787 \\
& 8 & 2.970045457888 & 12.546986400829 & -1.001639862160 & 0.000000000022 & 0.176571464280 \\
$5-2$ & 1 & 1.054655818598 & 12.566721344965 & -1.001877081522 & 0.000000000692 & 1.395140016716 \\
& 2 & 2.631700119053 & 12.565144225295 & -1.001295014690 & 0.000000000406 & 0.608148097048 \\
\hline
\end{tabular}


For the parametrisation of the $p-q$ resonant families of orbits, we have used the Jacobi constant, taking into account the behaviour displayed in Figure 2. As it can be seen, for each value of the Jacobi constant there are two admissible values of the polar angle in $\psi \in(-\pi / 2,3 \pi / 2)$. According to this, we have divided each family in two parts: the left hand part, for $\psi \in(-\pi / 2, \pi / 2)$ and the right hand part, for $\psi \in(\pi / 2,3 \pi / 2)$.

In Figures 3 and 4 we have represented the results of the numerical refinements for three families: $p-q=1-2,2-3$ and 3-2. In all the figures we have represented the value of $C_{J}$ in the $x$-axis and the modulus of the residual velocity, $\Delta v=\sqrt{\left(\dot{x}_{f}-\dot{x}_{i}\right)^{2}+\left(\dot{y}_{f}-\dot{y}_{i}\right)^{2}}$, in the $y$-axis. As it can be clearly seen, for all the families there are several isolated values of $C_{J}$ at which the value of $\Delta v$ goes to zero. For these values of the Jacobi constant we have been able to find periodic orbits whose initial conditions are given in Tables I and II. Some of these periodic orbits have been represented in Figures 5 and 6 , respectively.
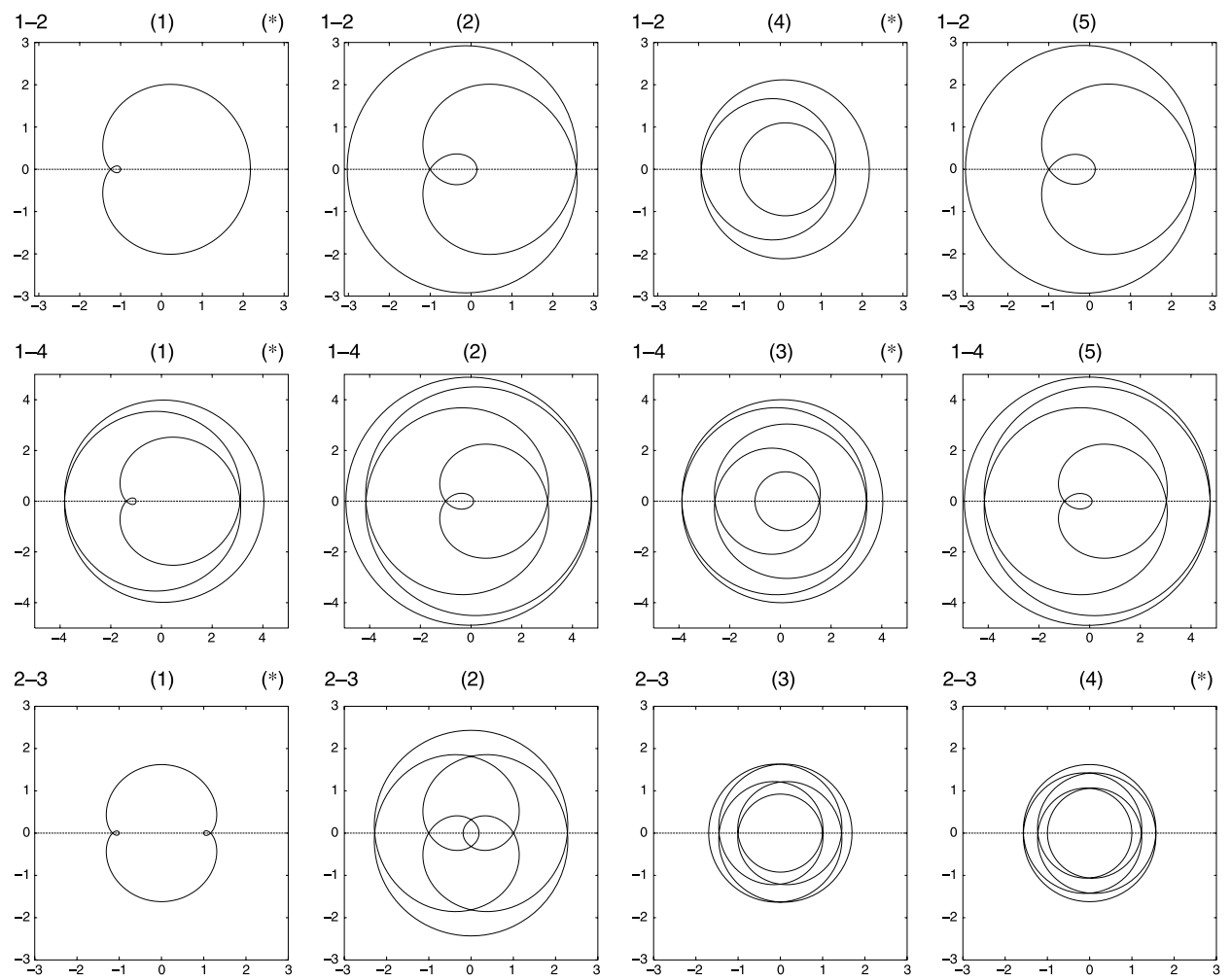

Figure 5. Periodic orbits computed for several $p-q$ resonances with $p<q$. On the top of each figure the values of $p-q$ and the number of the orbit, according to Table I, are given. The orbits with $\left(^{*}\right)$ are close to the $p-q$ resonance. 

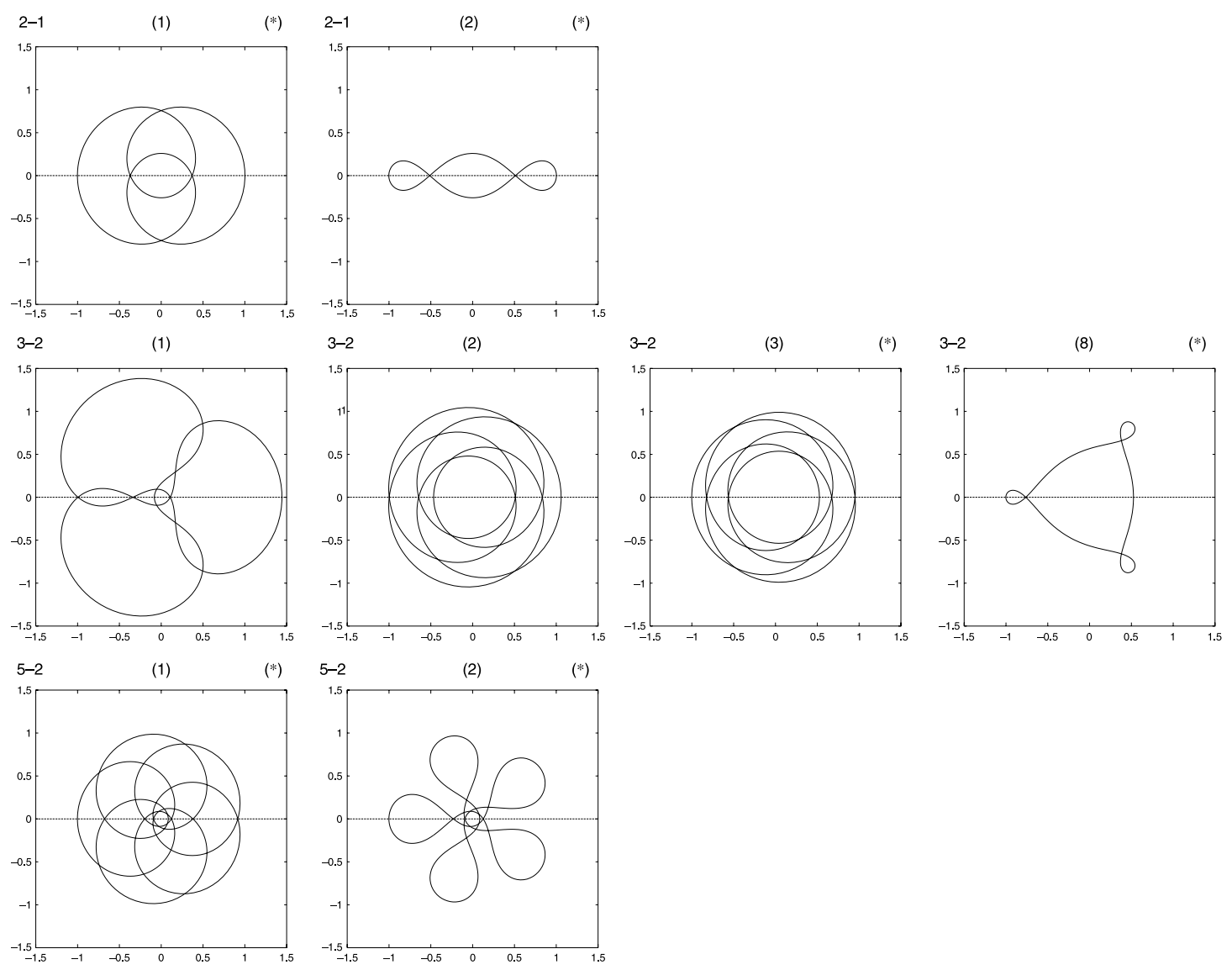

Figure 6. Periodic orbits computed for several $p-q$ resonances with $p>q$. On the top of each figure the values of $p-q$ and the number of the orbit, according to Table II, are given. The orbits with $(*)$ are close to the $p-q$ resonance. 
Next, there are several comments respect to the results obtained:

- We have used double precision to compute all the orbits and we have asked for periodicity with an error of $10^{-11}$. The integration was done using a RungeKutta-Fehlberg of order 7-8 and local truncating error less than $10^{-13}$.

- The points marked on the $C_{J}(\psi)$ curves (Figure 2) are those for which he have found periodic orbits. For different admissible pairs $p-q$, the initial conditions, the Jacobi constant and the period of these orbits are in Tables I and II. Some of these orbits are shown in Figures 5 and 6. We have not included all the orbits because the orbit corresponding to a point on the right hand of the $C_{J}(\psi)$ curve and the orbit corresponding to a point on the left hand with the same value of the Jacobi constant looks like similar: one is a direct motion and the other is a retrograde motion.

- For a fixed pair $p-q$ we have found two kinds of orbits: orbits with an intermediate passage near the small primary between the two encounters that define the resonance, and orbits which do not approach the small primary apart from these two encounters. Although the first ones are obtained from initial conditions of $p-q$ resonant orbits, they are not $p-q$ resonant: when the third body meets $M$ after leaving its neighbourhood, the small body has not complete $q$ revolutions around the centre of masses of the two primaries. These orbits are marked in Figure 2 with crosses. The points marked by triangles in the figure correspond to orbits close to $p-q$ resonant orbits. We can observe that they correspond to points near the maximum or the minimum value of the Jacobi constant for each $p-q$ family: the values $C_{J_{1}}$ and $C_{J_{2}}$ defined in (4).

- All the periodic orbits founded are symmetric respect to the synodical $x$ axis. In particular, the orbits close to a $p-q$ resonance have a perpendicular cross with the horizontal axis near the small primary (see Figures 5 and 6). The values of the Jacobi constant for these orbits are near the values $C_{J_{i}}$ and, from (5), $\sin \psi= \pm 1$, which implies $\dot{x}_{0}=0$. In Tables I and II, the initial conditions given do not coincide necessarily with the perpendicular cross.

\section{Symmetric Periodic Second Species Solutions}

As has been mentioned in Section 1, the SSSs have as natural limit, when $\mu \rightarrow 0$, the so called orbits with consecutive collisions (OCC). These families can be described analytically and used as generating families of SSS. A description of the families of arcs of consecutive collision can be founded in Bruno (1994), as well as the families of periodic solutions generated by them. In this section we will extend numerically some families of symmetric OCC to a value of the mass parameter equal to $0.30401879 \times 10^{-5}-($ Earth + Moon $)-$ Sun mass ratio - which is very close to the one used in the preceding section $10^{-6}$.

Hénon classifies the OCC in several families denoted by $A_{0}, A_{i}, B_{i}$ and $C_{i j}$ for $i, j \in \mathbb{N}$ and represents each family by its characteristic curve in the $(\tau / \pi, \eta / \pi)$ 


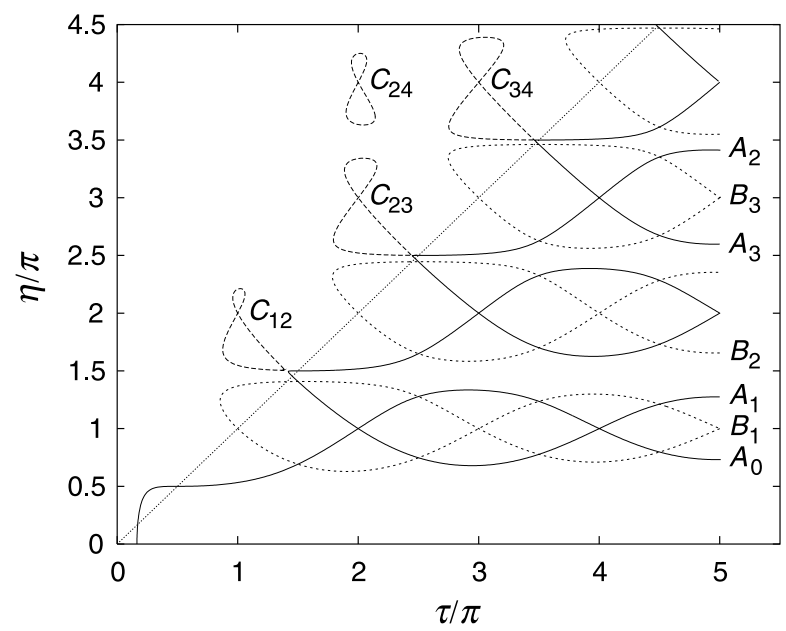

Figure 7. Hénon diagram of the characteristic curves of the families of orbits of consecutive collisions with $\tau<5 \pi$ and $\eta<4.5 \pi$.

plane, where $\tau$ is the true anomaly of $P$ at the collision and $\eta$ its eccentric anomaly at the same point (see Figures 1 and 7). The families can be described as follows (see Hénon, 1968):

- the family $A_{0}$ begins at some value of $\tau$ and it continues for values of $\tau$ growing up to $\infty$, while $\eta / \pi \leqslant 1.5$.

- in the families $A_{i}$, for $i \in \mathbb{N}, \tau$ decreases from $\infty$ to a minimal value near the intersection with the line $\tau=\eta$, and then increases up to $\infty$ again. Each $A_{i}$ intersects with $A_{i-1}$ and $A_{i+1}$.

- the behaviour of the families $B_{j}$ are similar to the families $A_{i}$, but each family only intersects with itself.

- the families $C_{i j}$ are closed curves around the double point $\tau / \pi=i$ and $\eta / \pi=$ $j$, for $i, j$ such that $1<j / i<2 \sqrt{2}$.

Here we will use the same notation both for the generating families and for their continuation for $\mu>0$. In this last situation we will denote them by $A_{i}^{\mu}, B_{i}^{\mu}$ and $C_{i j}^{\mu}$.

For small values of $\mu$, the characteristic curves of $A_{i}^{\mu}, B_{i}^{\mu}$ and $C_{i j}^{\mu}$ are close to the characteristic curves of generating families $(\mu=0)$, except in the vicinity of the bifurcation points, where the characteristic curve of the family of SSS breaks (see Figures 8 and 9). These points correspond to the points where the corresponding family of generating orbits intersects another family. In Hénon's diagram, in the $(\tau / \pi, \eta / \pi)$ plane, bifurcation orbits correspond to the following points:

- some intersections between the families $A_{0}, A_{i}$ or $B_{j}$ with the line $\tau=\eta$, for any $i, j \in \mathbb{N}$;

- the intersections between the families $A_{i}$ and $A_{i-1}$ for any $i \in \mathbb{N}$, which occur at points $(2 k, i)$, for $2 k>i$ and $i$ odd and $(2 k+1, i)$, for $2 k+1>i$ and $i$ even; 

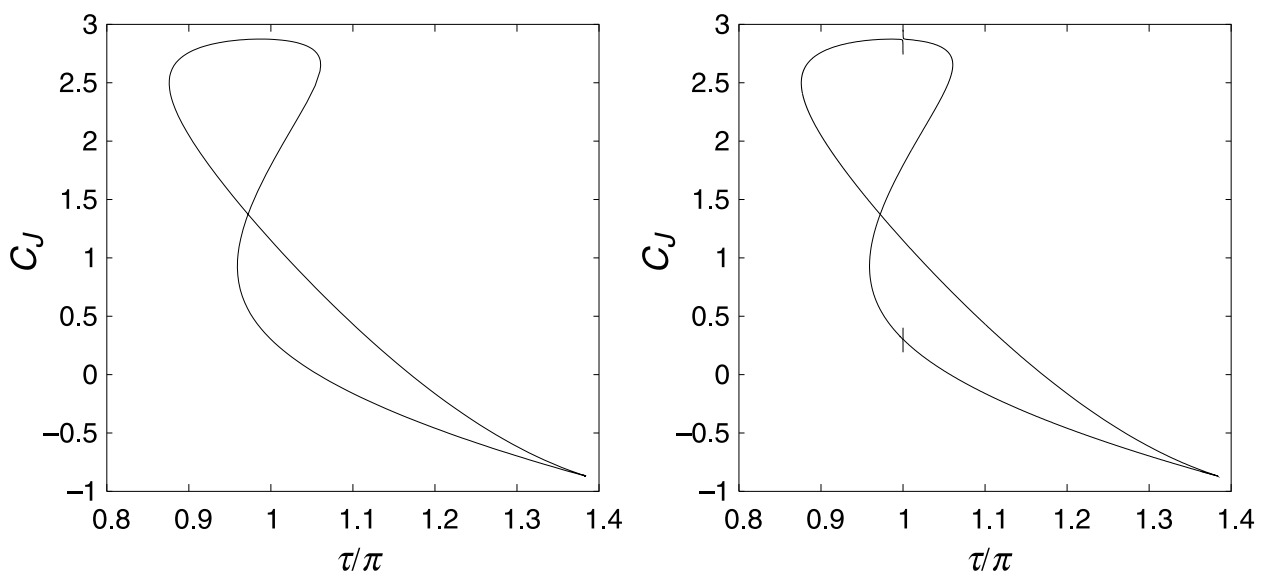

Figure 8. Characteristic curves, $\left(\tau / \pi, C_{J}\right)$, of the family $C_{12}(\mu=0$, on the left) and the family $C_{12}^{\mu}\left(\mu=0.30401879 \times 10^{-5}\right.$, on the right). The points of discontinuity (for $\left.\mu>0\right)$ correspond to bifurcation orbits represented by $\tau / \pi=1$ and $\eta / \pi=2$ in Hénon's diagram.
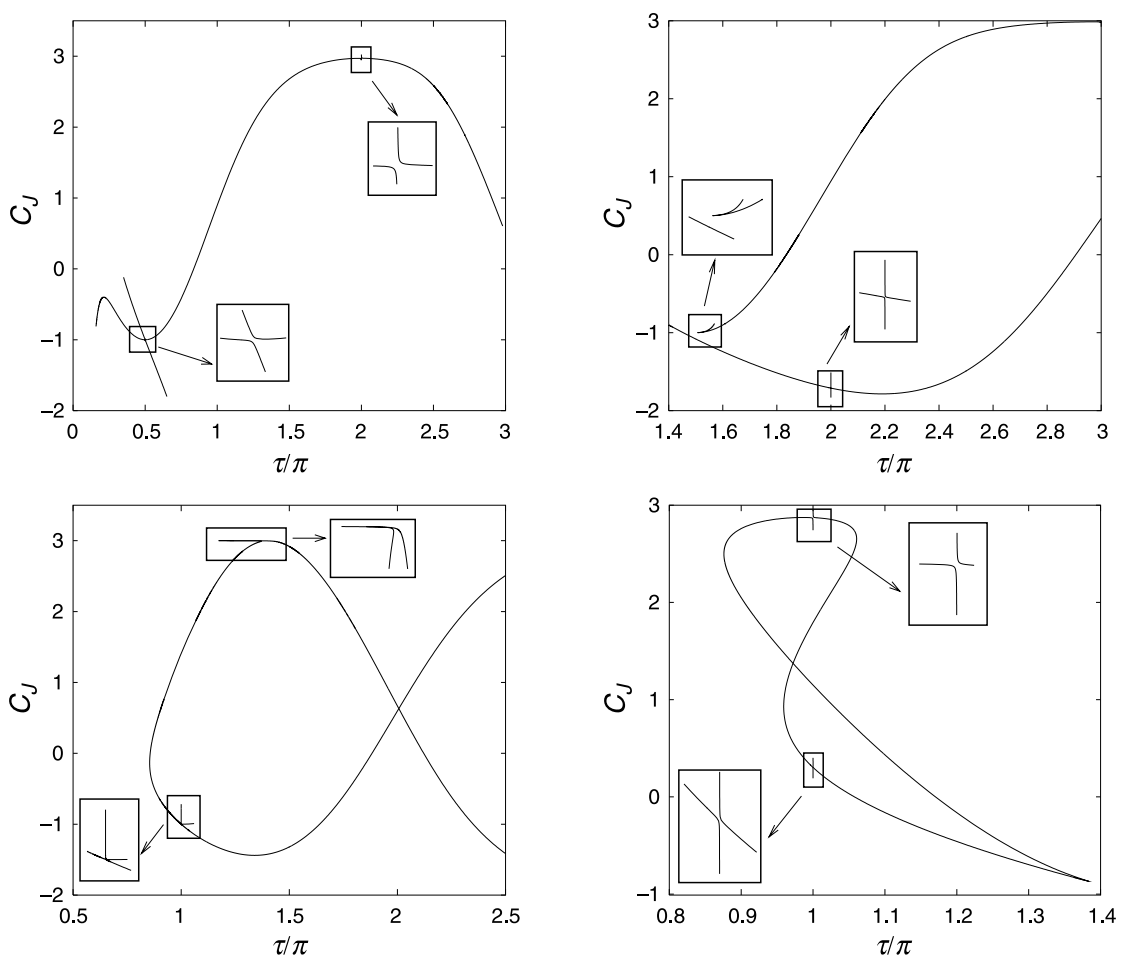

Figure 9. Characteristic curves of the families $A_{0}^{\mu}$ (top left figure), $A_{1}^{\mu}$ (top right figure), $B_{1}^{\mu}$ (bottom left figure) and $C_{12}^{\mu}$ (bottom right figure) for $\mu=0.30401879 \times 10^{-5}$. The points where the characteristic breaks correspond to bifurcation orbits in Hénon's diagram. 
- the intersections between $B_{j}$ with itself for any $j \in \mathbb{N}$, which occur at points $(2 k+1, j)$, for $2 k+1>j$ and $j$ odd and at points $(2 k, j)$ for $2 k>j$ and $j$ even; - the intersections between $C_{i j}$ with itself, which occur at points $(i, j)$ for any $i<j<2 \sqrt{2} i$.

Consider a periodic $p-q$ resonant orbit, so $p, q \in \mathbb{N}$ are relatively prime with $p / q<2 \sqrt{2}$. When $\mu \rightarrow 0$, the generating orbit will be an orbit with consecutive collisions with mean motion $p / q$ and semi-major axis $(q / p)^{2 / 3}$. This implies that $\tau / \pi=q$ and $\eta / \pi=p$ (between two consecutive collisions, $M$ does $q$ revolutions while $P$ does $p$ revolutions). So, the generating orbit will be represented by the point $(q, p)$ in Hénon's diagram. On the other hand, and according to the above comments, the points $(q, p)$ will correspond to bifurcation orbits. If $q<p$, then the point $(q, p)$ will belong to the family $C_{q p}$. If $q>p$, the point $(q, p)$ will belong to the intersection between two families $A$ 's or between a family $B$ with itself, depending on the parity of $p$ and $q$ as follows:

- if $p$ is even and $q$ is odd, or vice versa, then $(q, p)$ will belong to $A_{p-1} \cap A_{p}$; - if $p$ and $q$ are odd, then $(q, p)$ will belong to $B_{p} \cap B_{p}$.

Now, let us consider any of the symmetric periodic orbits obtained in Section 3. We would like to identify the family of SSSs to which it belongs. The $p$ and $q$ resonance values give the two generating families whose intersection produces the bifurcation orbit. If $p>q$, then the generating orbit belongs to the $C_{q p}$ family, and the periodic orbit to $C_{q p}^{\mu}$. If $p<q$ and both are odd, then the generating orbit belongs to the family $B_{p}$, so the periodic orbits will belong to $B_{p}^{\mu}$. If they are of different parity, then the generating orbit belongs to the intersection of $A_{p}$ and $A_{p-1}$. In order to identify to which family $\left(A_{p}^{\mu}\right.$ or $\left.A_{p-1}^{\mu}\right)$ belongs the p.o., we need to use the Jacobi constant and/or other parameters (such as its initial conditions or the stability parameters). For example,

— in the 1-2 resonance we have found periodic orbits for values of $C_{J}$ near 2.97 and -1.71 (see Table I). As $p=1$, then the generating orbit will be on the intersection between $A_{1}$ and $A_{0}$ at $\tau / \pi=q=2$. As we can see in Figure 9 the bifurcation at this point corresponds to values of $C_{J}$ near 3 for the family $A_{0}^{\mu}$ and to values of $C_{J}$ near -2 for the family $A_{1}^{\mu}$. This allows us to identify the family corresponding to each orbit.

- in the 2-3 resonance, we have found periodic orbits for values of $C_{J}$ near 2.99 and -0.39 (Table I). As $p=2$ and $q=3$, then the generating orbit will be on the intersection between $A_{2}$ and $A_{1}$ at $\tau / \pi=3$. Following the behaviour of the $C_{J}$ along each family, we can conclude that the p.o. with $C_{J}$ near 3 belongs to $A_{1}^{\mu}$ and the p.o. with $C_{J}$ negative, to the family $A_{2}^{\mu}$.

- in the 2-1 resonance, we have found periodic orbits for values of $C_{J}$ near 2.87 and 0.305 (Table II). Both belong to $C_{12}^{\mu}$ family, and they correspond to the bifurcations showed in Figure 9. 


\section{Conclusions}

In this paper we have computed planar SSSs of the RTBP which, at the same time, are of a rather special kind of periodic $p-q$ resonant orbits. When the resulting orbits are symmetric, the corresponding generating OCC have been identified. Some bifurcation diagrams, associated with the families of OCC: $A_{0}, A_{1}, B_{0}, B_{1}$ and $C_{12}$, are also given.

\section{Acknowledgements}

The researchers have been supported by grant CIRIT 2001SGR-70 (Catalonia) and the second author by grant DGICYT BFM2000-805 (Spain).

\section{References}

Barrabés, E. and Gómez, G.: 2002, 'Spatial $p-q$ resonant orbits of the RTBP', Celest. Mech. Dyn. \& Astr. 84(4), 387-407.

Barrabés, E. and Gómez, G.: 2003, 'Three dimensional $p-q$ resonant orbits close to second species solutions', Celest. Mech. \& Dyn. Astr. 85(1), 145-174.

Belbruno, E. and Marsden, B.: 1997, 'Resonance hopping in comets', Astron. J. 113(4), 1433-1444.

Bruno, A. D.: 1994, The Restricted 3-Body Problem: Plane Periodic Orbits (engish translation of Bruno 1990). De Gruyter Expositions in Mathematics 17, Walter de Gruyter, Berlin, New York.

Font, J., Nunes, A. and Simó, C.: 2002, 'Consecutive quasi-collisions in the planar circular RTBP', Nonlinearity 15(1), 115-142.

Gómez, G. and Ollé, M.: 1986, 'A note on the elliptic restricted three-body problem', Celest. Mech. 39(1), 33-55.

Hénon, M.: 1968, 'Sur les orbites interplanetaires qui rencontrent deux fois la terre', Bull. Astron., III. Ser. 3, 377-402.

Hénon, M.: 1997, Generating Families in the Restricted Three-Body Problem, Springer-Verlag, Berlin.

Koon, W. S., Lo, M. W., Marsden, J. E. and Ross, S. D.: 2001, 'Resonance and capture of Jupiter comets', Celest. Mech. \& Dyn. Astr. 81(1-2), 27-38.

Poincaré, H.: 1899, Les Méthodes Nouvelles de la Mécanique Céleste, Gauthier-Villars, Tome III.

Yen, C. L.: 1985, 'Ballistic Mercury orbiter mission via Venus and Mercury gravity assist', AAS/AIAA Astrodynamics Specialist Conference, Paper AAS 85-346. 